\title{
MAGNETIZATION VERSUS HEAT TREATMENT IN RAPIDLY SOLIDIFIED NdFeB
} ALLOYS

\author{
M.T. CLAVAGUERA-MORA, M.D. BARÓ, S. SURIN̄ACH, J.A. DIEGO
}

Física de Materials, Departament de Física, Universitat Autònoma de Barcelona, 08193 - Bellaterra, Spain

\section{N. CLAVAGUERA}

Departament d'Estructura i Constituents de la Matèria, Universitat de Barcelona, Diagonal 647, 08028 - Barcelona, Spain

\begin{abstract}
NdFeB melt spun amorphous or partially amorphous alloys of four compositions were prepared. Their crystallization kinetics induced by thermal treatment was studied by differential scanning calorimetry and scanning and transmission electron microscopy. Scanning electron microscopy demonstrated that heterogeneous nucleation occurs preferentially at the ribbon surface which was in contact with the wheel. The explicit form of the kinetic equation that best describes the first stage of crystallization under high undercooling conditions was obtained for each alloy. From the crystalization results, the lower part of the experimental time-temperature-transformation curves was deduced for each alloy and extrapolated up to the high temperature limit of their validity. Microstructural observations showed a typical size of the microcrystals obtained by heat treatment of $\sim 100 \mathrm{~nm}$. From the magnetic properties measured with a vibrating sample magnetometer, the same magnetic behaviour of partially crystallized alloys is observed regardless of the temperature of annealing provided the same crystallization fraction, $x$, is achieved, at least for small values of $x$ (typically 10\%).
\end{abstract}

\section{INTRODUCTION}

The development of the rapid solidification route to produce permanent magnets began in the late 1978 when, for the first time, coercivities in excess of $15 \mathrm{kOe}$ were obtained in melt-spun $\mathrm{Fe}-\operatorname{Pr}(\mathrm{BSi})$ alloys which were subsequently crystallized [1]. This discovery opened a new horizon for magnetic materials research and revolutionized the world of permanent magnets [2]. It is now well established that in the melt spinning route for the production of $\mathrm{NdFeB}$ magnets the control of the final grain size is crucial in the development of the hard magnetic properties.

The aim of this study was to investigate morphologically and calorimetrically the crystallization kinetics of some amorphous melt-spun NdFeB alloys and to correlate it with the magnetic properties. Some preliminary results are presented here.

\section{EXPERIMENTAL}

Master alloys of NdFeB were provided by $\mathrm{Dr}$. $\mathrm{S}$. Sattelberger from Gesellschaft für Elektrometallurgie, Nurnberg. The compositions of the as-cast materials used are indicated in Table 1. Melt-spun alloys were obtained by quenching the molten materials on the surface of a rapidly spinning $(\sim 30 \mathrm{~m} / \mathrm{s})$ cooper wheel under a helium atmosphere. Melt spun alloys \#1 to \#3 were obtained as a mixture of short ribbons and pellets. The X-ray diffraction patterns of the pellets showed a small degree of crystallinity on them (less than 10 vol\%). Melt-spun alloy \#4 was obtained in normal ribbon form. X-ray diffraction confirmed the amorphous state of all the ribbons (within the experimental limit of detection of crystals).

Differential scanning calorimetric (DSC) measurements were performed in a computerized Perkin Elmer DSC-2 equipment in both isothermal and continuous heating regimes (for more details see ref. [3]) on the melt-spun ribbons. Isothermal anneals were performed in the range $800-850 \mathrm{~K}$ by heating the sample $(\sim 10 \mathrm{mg})$ from room temperature to the annealing temperature at a rate of $360 \mathrm{k} / \mathrm{min}$. The interval of crystallization temperatures scanned in continuous heating experiments extended from 830 to $980 \mathrm{~K}$ with heating rates in the range $10-320 \mathrm{~K} / \mathrm{min}$. To analyse the crystallization kinetics, both the crystallized fraction, $x$, and the transformation rate, $\mathrm{dx} / \mathrm{dt}$, at a given time or temperature, were determined following the current procedure [3]. The uncertainty in temperature was $\pm 3 \mathrm{~K}$, those on $x$ and $d x / d t$ (in the range $0.1 \leq x \leq 0.9$ ) were less than $5 \%$

Microstructural observations were carried out on thin foils prepared by ion beam milling with a Philips EM 300 transmission electron microscope (TEM) at the Birmingham University (Prof. I.R. Harris) and on fresh fracture surfaces with a Hitachi S-570 SEM at the Autonomous University of Barcelona. The magnetic properties were measured with a vibrating sample magnetometer (Birmingham University) in fields up to $14 \mathrm{kOe}$.

\section{RESULTS AND DISCUSSION}

The DSC curves for the four amorphous ribbons obtained at a heating rate of $40 \mathrm{~K} / \mathrm{min}$ are compared in fig. 1. One to four crystallization peaks, of ten overlapping, appear on heating. The general form of the exothermic peaks agrees with that published for melt-spun alloys of similar compositions $[4,5]$

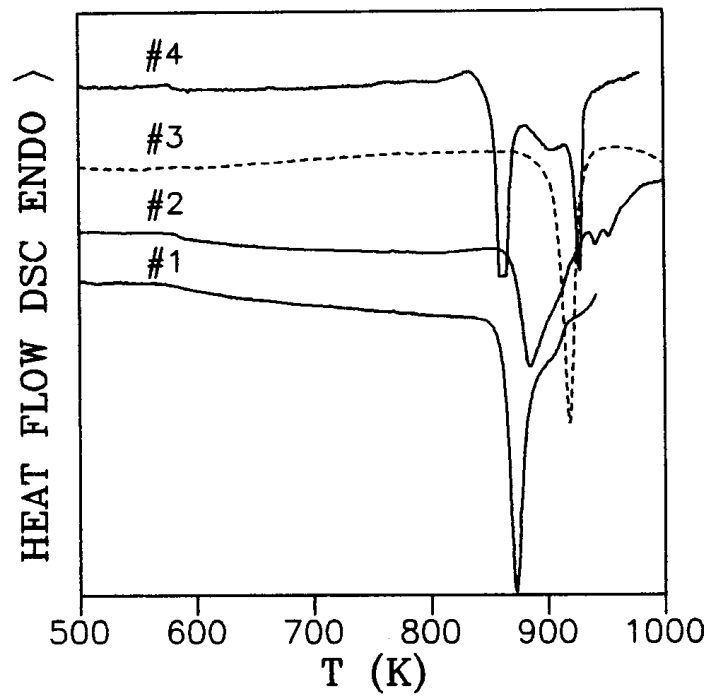

Fig. 1.- DSC scans (arb. units) for the melt spun ribbons studied (scan rate $40 \mathrm{~K} / \mathrm{min}$ ). 
As regards to fig. 1 , the most stable material versus crystallization is alloy \#3. It shows no Curie transition in the as-quenched state in the temperature interval 400-640 $\mathrm{K}$ (neither do amorphous alloys \#1, \#2). Once crystallized it shows a Curie transition at $648 \mathrm{~K}$ which can be ascribed to the metastable $\mathrm{Nd} 2 \mathrm{Fe} 23 \mathrm{~B} 3$ compound [6]. Next material stable versus crystallization (at that heating rate) is alloy $\# 2$. In the crystallized state it shows a Curie transition at $579 \mathrm{~K}$. This Curie temperature corresponds to the offstoichiometric $\mathrm{Nd} 2 \mathrm{Fe} 14 \mathrm{~B}$ compound $[7,8]$. In decreasing stability versus crystallization, next material is alloy \#1. The obverved Curie transition at $575 \mathrm{~K}$ for the crystallized material was ascribed to off-stoichiometric Nd2Fe14B. Finally, alloy \#4 is the least stable versus crystallization at these temperatures. A clear Curie transition of the amorphous alloy appears at $577 \mathrm{~K}$ while the Curie temperature of the crystallized material is $765 \mathrm{~K}$. The value expected for metastable Fe3B compound would be $810 \mathrm{~K}$ [9]. The glass transition could also be observed for this alloy and it occurs at a temperature $\mathrm{Tg}=803 \mathrm{~K}$.

To study the crystallization processes we assume that in the limited temperature interval experimentally accessible by DSC measurements, each crystallization exotherm has a kinetics that follows a general equation of the form

$$
d x / d t=K(T) f(x)
$$

We further assume that the rate constant follows an Arrhenius behaviour

$$
\mathrm{K}(\mathrm{T})=\mathrm{K}_{0} \exp (-\mathrm{E} / \mathrm{kT})
$$

with Ko the pre-exponential factor and $E$ the apparent activation energy.

Since the crystallization peaks were somewhat overlapping at various heating rates, the apparent activation energy was deduced by the Kissinger method from the continuous heating data. The values obtained for $E$ are quoted in Table 1. They are very similar to those published for other $\mathrm{NdFeB}$ alloys [10].

With isothermal measurements at rather low temperatures the first crystallization peak alone occured. Therefore, the value of the function $f(x)$ for this first peak could be measured, except for a constant factor [11]. Fig. 2 shows the experimental values of $\ln (d x / d t)$ versus $\ln (1-x)$ obtained under isothermal conditions for the four alloys. From these results $\operatorname{Kof}(\mathrm{x})$ was deduced for each alloy.

More interestingly, the integrated form of equation (1) under isothermal conditions gives

$$
\begin{array}{ll} 
& g(x)=\int d x / f(x)=K(T) t \\
\text { or } & g(x) / K_{0}=t \exp (-E / R T)
\end{array}
$$

As a consequence, isothermal measurements on each alloy provided the value of $g(x) / K_{0}$ with $x$ in the range $0.1-0.9$ for the first crystallization peak. Note that $x=1$ applied to the first crystallization peak

\begin{tabular}{|c|c|c|c|c|c|}
\hline \multirow{2}{*}{ Alloy } & \multicolumn{3}{|c|}{ Composition(at. \%) } & \multirow{2}{*}{ peak } & \multirow{2}{*}{$E(e V)$} \\
\hline & $\mathrm{Nd}$ & $\mathrm{Fe}$ & $\mathrm{B}$ & & \\
\hline \#1 & 13 & 82.2 & 4.8 & first & 2.99 \\
\hline \multirow[t]{4}{*}{$\# 2$} & 13 & 79 & 8 & first & 3.21 \\
\hline & & & & second & 3.65 \\
\hline & & & & third & 4.73 \\
\hline & & & & fourth & 5.18 \\
\hline$\# 3$ & 8.5 & 80.5 & 11 & first & 4. 54 \\
\hline \multirow[t]{2}{*}{$\# 4$} & 3 & 77 & 20 & first & 5.91 \\
\hline & & & & third & 3.95 \\
\hline
\end{tabular}

TABLE 1

COMPOSITION OF THE ALLOYS AND APPARENT ACTIVATION ENERGY OF EACH EXOTHERMIC CRYSTALLIZATION PEAK

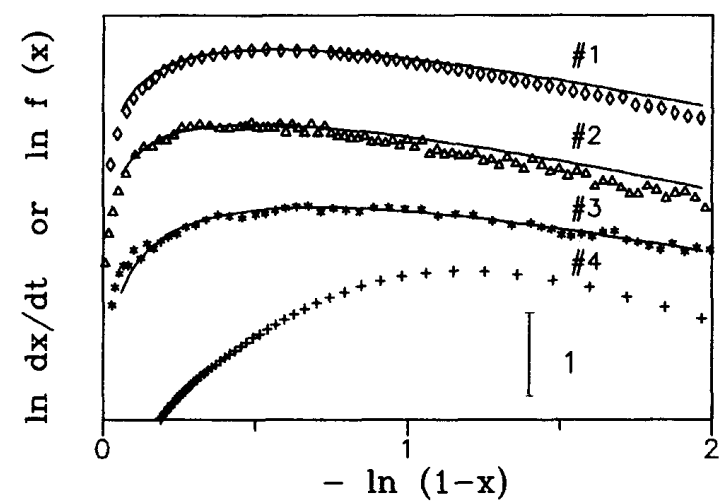

Fig. 2. - Experimental values of $\ln (d x / d t)$ vs. $-\ln (1-x)$ obtained under isothermal conditions for the four alloys. When a theoretical model gives good agreement with the experimental data it is shows as a solid line (see ref. [11]).

gives the completion of this peak, but other crystallization processes can still occur (mostly on increasing the temperature)

The DSC technique is very powerful for determining the classical time-temperature-transformation ( $T-T-T$ ) curves. Effectively, equation (4), when represented as the temperature versus time needed to crystallize a fixed fraction $x$ of material, gives the $T-T-T$ curve. Then the knowledge of the explicit form of $f(x)$ (or $g(x))$, Ko and $E$ allows construction of the $T-T-T$ curve. The resulting $T-T-T$ curves for a crystallized fraction of $x=0.1$ are shown in fig. 3. The analysis of this figure shows that above $830 \mathrm{~K}$ the time needed to crystallize an alloy decreases in the sequence \#3, \#2, $\# 1$ and $\# 4$, or in other words, this is the sequence of decreasing stability against crystallization (these are the results observed in the continuous heating DSC curves of fig. 1). But bellow $830 \mathrm{~K}$ the ordering of the sequence changes and alloy \#4 becomes more stable than \#1 and \#2 on decreasing the temperature.

To elucidate the microstructure versus heat treatment, the four amorphous alloys were subjected to annealings of $10 \mathrm{~min}$ and 1 hour at the temperatures needed to get $x=0.1$ (see fig. 3 ). Fig. 4 shows the fine microstructure of crystallized portions of heat treated (HT) alloy \#2. Similar results were obtained for the other alloys. At low annealing temperature

\section{TTT curves $\quad(x=0.1)$}

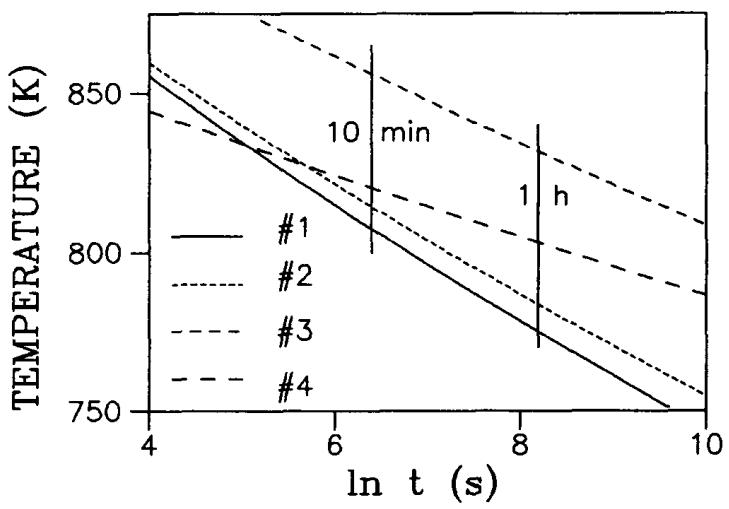

Fig. 3. - Predicted low temperature part of the $T-T-T$ curves for the four NaFeB alloys studied. 


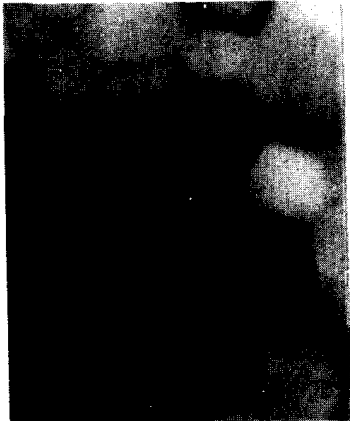

a

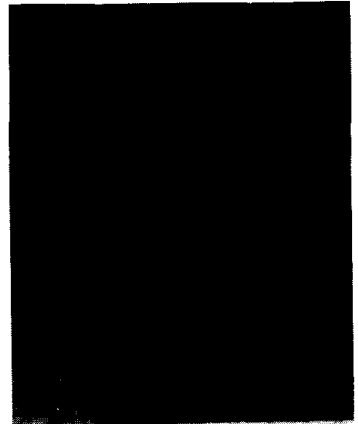

$\overline{50 \mathrm{~nm}}$
Fig. 4. - TEM micrographs from thin foils of alloy \#2 showing the $\mathrm{Nd2Fe} 14 \mathrm{~B}$ equiaxed grains. a) after $1 \mathrm{~h}$ at $780 \mathrm{~K}$; b) After $10 \mathrm{~min}$ at $810 \mathrm{~K}$.

Fig. 5. - SEM image of the fracture of alloy \#2 (near the free surface) after annealing $15 \mathrm{~min}$ at $1000 \mathrm{~K}$.
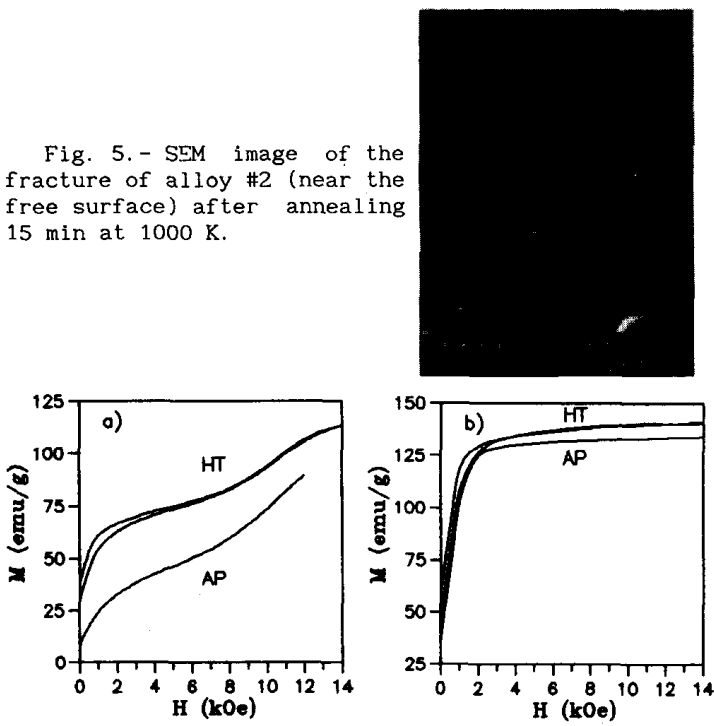

Fig. 6. - Magnetization curves for: a) alloy \#1; b) alloy \#3.

(fig. 4a) the typical size of the crystals is $\sim 50 \mathrm{~nm}$ and it decreases to a smaller size at the high annealing temperature ( $f$ ig. $4 \mathrm{~b}$ ). When annealing the melt-spun material at higher temperatures the whole crystallization process occurs and the typical size of the crystals remains $\sim 100 \mathrm{~nm}$. As an example, fig. 5 shows a SEM image of a fresh fracture of alloy \#2 after annealing for $15 \mathrm{~min}$ at $1000 \mathrm{~K}$. This figure depictes a portion of the ribbon near the free surface and typical sizes of the crystalline grains are $\sim 50 \mathrm{~nm}$. Systematic observations showed that the microstructure of the material once crystallized at $1000 \mathrm{~K}$ is refined from the wheel surface (size $\sim 200 \mathrm{~nm}$ ) towards the free surface.

The magnetization curves for as-prepared (AP) and heat-treated (HT) alloys \#1 and \#3 are show in fig. 6 . The large slope of the magnetization at $14 \mathrm{kOe}$ for alloy \#1 indicates that the sample is far from saturation; a soft (amorphous) and a hard (crystalline) phase coexist whose magnetization improves on heat treatment, probably because it results in a small increase of the degree of crystallinity and also a relaxation of the amorphous phase. Similar behaviour was found for alloy \#2. Alloys \#3 and \#4 have a magnetization curve quite different (shown for alloy
\#3 in fig. 5b). The fact that the crystallization product is $\mathrm{Nd2Fe} 3 \mathrm{~B} 3$ may explain this behaviour. One important feature for all the alloys studied is that the magnetic behaviour is the same regardless of the heat treatment (temperature or time) provided the same crystallized fraction is achieved. This result has to be confirmed for higher values of $\mathrm{x}$ and more wide temperature (or time) intervals.

\section{CONCLUSIONS}

Amorphous melt-spun $\mathrm{NdFeB}$ ribbons were prepared under controlled conditions and their crystallization kinetics was studied both morphologically and calor imetrically. DSC analysis reveals that crystallization proceeds generally in various stages. Microscopic analysis shows that the crystalline size of the heattreated material is about $50 \mathrm{~nm}$. The crystallization kinetic study allowed construction of part of the T-T-T curve for each alloy. Preliminary results indicate that the magnetic properties of the partially crystallized materials are correlated with the crystalline fraction achieved by heat treatment rather than with the temperature or time of annealing independently. This result agrees with the fact that the crystalline size is rather insensitive to the temperature of annealing.

\section{ACKNOWLEDGEMENTS}

The authors wish to thank Prof. I.R. Harris for encouraging discussions and experimental facilities.

This work was supported by EURAM [contract No. MA1E. 0061. C(H)] and by CICYT [project No. MAT88-0314] which are acknowledged. One of the authors, J.A.D. acknowledges support from CIRIT [AR/89].

\section{REFERENCES}

[1] G.C. Hadjipanayis, R.C. Hazelton and K. R. Lawless, "New iron-rare-earth based permanent magnets materials", Appl. Phys. Lett., vol. 43, pp. 797-,1983.

[2] K.H.J. Buschow, "New permanent magnet materials", Mater. Sci. Reports, vol. 1, pp. 1-64, 1986.

[3] S. Suriñach, M. D. Baró, M. T. Clavaguera-Mora and N. Clavaguera, "Kinetic study of isothermal and continuous heating crystallization in GeSez-GeTeSb2Te3 alloy glasses", J. Non-Cryst. Solids, vol. 58, pp. 209-217, 1983.

[4] A. J.W. Ogilvy, G.P. Gregan and H. A. Davies, in "Nd-Fe Permanent Magnets - Their present and future applications", I.V. Mitchell ed., CEE, Brussels, 1985, pp. 93-97.

[5] A. Jha, H. A. Davies and R.A. Buckley, "Glass forming ability and kinetics of crystallisation of rapidly quenched Nd-Fe-B alloys", J. Magn. and Magn. Mater., vol. 80, pp. 109-114, 1989.

[6] K.H.J. Buschow, D.B. de Mooij and H.M. van Noort, "Properties of metastable ternary compounds and amorphous alloys in the $\mathrm{Nd}-\mathrm{Fe}-\mathrm{B}$ system", J. Less-Common Met., vol. 125, pp. 135-146, 1986.

[7] K.H. J. Buschow, D.B. de Mooij, J.L.C. Daams and H.M. van Noort, "Phase relationships, magnetic and crystallographic properties of $\mathrm{Nd}-\mathrm{Fe}-\mathrm{B}$ alloys", J. Less-Common Met., vol. 115 , pp. 357-366, 1986.

[8] Y.C. Chuang, C. H. Wu, Y.C. Chang and L. KaO, "Structure and magnetic properties of some $\mathrm{Nd}-\mathrm{Fe}-\mathrm{B}$ alloys", J. Less-Common Met., vol. 147, pp. 113-120, 1989.

[9] C.L. Chien, D. Musser, E.M. Gyorgy, R. C. Sherwood, H.S. Chen, F.E. Luborsky and J.L. Walter, "Magnetic properties of amorphous FexB100-x $(72 \leq x \leq 86)$ and crystalline FesB", Phys. Rev. B, vol. 20, pp. $283-295,1979$

[10] A. Jha and H.A. Davies, "Kinetics of crystallisation of rapidly quenched FeNdB alloys and its applications in the processing of permanent magnets", J. Non-Cryst. Solids, vol. 113, pp. 185-194, 1989.

[11] M. T. Clavaguera-Mora, M.D. Baró, S. Suriñach, N. Clavaguera, "Crystallization behaviour of some melt spun NdFeB alloys", J.Mat. Res., vol.6, 1990 (in press). 